Radiologe 2011 · 51:455

DOI 10.1007/s00117-011-2186-y

Online publiziert: 29. Mai 2011

(c) Springer-Verlag 2011

\author{
M.F. Reiser ${ }^{1}$ - D.-A. Clevert ${ }^{1} \cdot$ S. Delorme ${ }^{2}$ \\ ${ }^{1}$ Institut für Klinische Radiologie, Klinikum der \\ Ludwig-Maximilians-Universität, Campus Großhadern, München \\ ${ }^{2}$ Abt. E010 - Radiologie, Deutsches Krebsforschungszentrum (DKFZ), Heidelberg
}

\title{
Kontrastverstärkter Ultraschall und neue Techniken
}

Durch die jüngsten Entwicklungen im Bereich der Ultraschallgerätetechnik, insbesondere durch die Einführung der hochauflösenden Ultraschallköpfe, hat sich das Einsatzgebiet stetig erweitert und es konnten neue Fragestellungen in die tägliche Routine implementiert werden.

Als neue Anwendungsbereiche bieten sich z. B. Erkrankungen der Speicheldrüse oder eine Beurteilung der Mikrozirkulation des Muskelgewebes an.

Neben diesen klinischen Indikationen kann der kontrastverstärkte Ultraschall auch in der radiologischen Forschung eine nützliche Bereicherung darstellen. So können bei tierexperimentellen Untersuchungen zeitlich und räumlich hochaufgelöste Aufnahmen von Tumoren akquiriert und durch die Analyse der Kontrastmittelkinetik vor und nach Therapie funktionelle Informationen über den angiogenetisch-metabolischen Status des Gewebes gewonnen werden.

Dieses Schwerpunktheft von Der Radiologe zum Thema „Kontrastverstärkter Ultraschall und neue Techniken“ richtet sich an Ausbildungs- und Fachärzte der Radiologie sowie an interessierte Leser aus anderen Fachbereichen wie Internisten, Chirurgen und Neuroradiologen. Entsprechend der Zielsetzung von Der Radiologe sollen etablierte und in der $\mathrm{Zu}$ kunft in Praxis und Klinik viel versprechende Methoden und Techniken dargestellt und kritisch analysiert werden.

Wir bedanken uns bei den Autoren für ihre Mitwirkung an diesem Themenheft und hoffen damit, Ihr Interesse und Ihre Neugier zu wecken.

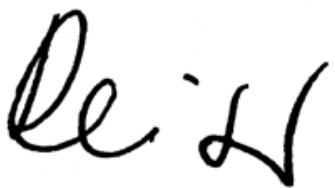

Prof. Dr. Maximilian Reiser

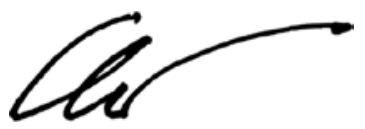

PD Dr. Dirk-André Clevert

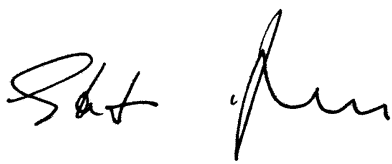

Prof. Dr. Stefan Delorme 